\title{
HPV Major Capsid Protein L1
}

National Cancer Institute

\section{Source}

National Cancer Institute. HPV Major Capsid Protein L1. NCI Thesaurus. Code C128312.

HPV major capsid protein L1 is encoded by the human papillomavirus L1 gene. This protein plays a role in viral capsid formation. 